\title{
Symmetric and asymmetric librations in extrasolar planetary systems: a global view
}

\author{
John D. Hadjidemetriou
}

Received: 15 November 2005 / Revised: 6 January 2006 /

Accepted: 20 February 2006 / Published online: 15 August 2006

(C) Springer Science+Business Media B.V. 2006

\begin{abstract}
We present a global view of the resonant structure of the phase space of a planetary system with two planets, moving in the same plane, as obtained from the set of the families of periodic orbits. An important tool to understand the topology of the phase space is to determine the position and the stability character of the families of periodic orbits. The region of the phase space close to a stable periodic orbit corresponds to stable, quasi periodic librations. In these regions it is possible for an extrasolar planetary system to exist, or to be trapped following a migration process due to dissipative forces. The mean motion resonances are associated with periodic orbits in a rotating frame, which means that the relative configuration is repeated in space. We start the study with the family of symmetric periodic orbits with nearly circular orbits of the two planets. Along this family the ratio of the periods of the two planets varies, and passes through rational values, which correspond to resonances. At these resonant points we have bifurcations of families of resonant elliptic periodic orbits. There are three topologically different resonances: (1) the resonances $(n+1): n,(2: 1$, $3: 2, \ldots),(2)$ the resonances $(2 n+1):(2 n-1),(3: 1,5: 3, \ldots)$ and $(3)$ all other resonances. The topology at each one of the above three types of resonances is studied, for different values of the sum and of the ratio of the planetary masses. Both symmetric and asymmetric resonant elliptic periodic orbits exist. In general, the symmetric elliptic families bifurcate from the circular family, and the asymmetric elliptic families bifurcate from the symmetric elliptic families. The results are compared with the position of some observed extrasolar planetary systems. In some cases (e.g., Gliese 876) the observed system lies, with a very good accuracy, on the stable part of a family of resonant periodic orbits.
\end{abstract}

Keywords Periodic orbits $\cdot$ Resonances $\cdot$ Extrasolar planetary systems

\footnotetext{
J. D. Hadjidemetriou ( $\varangle)$

Department of Physics, University of Thessaloniki,

54124 Thessaloniki, Greece

e-mail: hadjidem@auth.gr
} 


\section{Introduction}

In recent years, it was discovered that our Solar System is not the only planetary system in the universe. Up to the present there are 146 observed extrasolar planetary systems, with 18 of them having two or more planets. In many planetary systems with two planets close to each other, the two planets are in mean motion resonance. Examples are: HD 82943 (Israelian et al. 2001; Mayor et al. 2004), Gliese 876 (Marcy et al. 2001; Rivera and Lissauer 2001), at the 2:1 resonance and 55Cnc at the 3:1 resonance (Marcy et al. 2002). Some of these systems have large eccentricities and are evidently stable.

There are different approaches to the study of the dynamical evolution of a planetary system and on the mechanisms that stabilize the system, or generate chaotic motion and instability: Beaugé et al. (2003, 2006), Ferraz-Mello et al. (2003), Gozdjiewski et al. (2002), Ji et al. (2003a, b), Malhotra (2002), and Lee and Peale (2002, 2003). In these papers different methods have been applied as the averaging method, direct numerical integrations of orbits, or various numerical methods which provide indicators for the exponential growth of nearby orbits. In this way the regions where stable motion exists have been detected, in the orbital elements space.

The purpose of this paper is to present a global view of the structure of the phase space of a planetary system with two planets, moving in the plane, as obtained from the set of the families of periodic orbits. In this way, we can detect the regions where stable librations could exist. These will be the regions where a real planetary system could exist in nature. As we shall see, stable regions corresponding to elliptic orbits of the two planets with relatively large eccentricities are associated with mean motion resonances. We remark that stable motion could also exist far from resonances, if the eccentricities are small. This latter motion is close to a stable periodic orbit of the circular family of periodic orbits. We also remark that it is possible to have stable motion far from a periodic orbit, but we believe that in this latter case the two planets are not close to each other, so that their gravitational interaction is not very significant.

We found that the gravitational interaction between the planets is not negligible, even for very small planetary masses (compared to the mass of the star). In some cases it even dominates the attraction from the star. In this latter case the geometry of the planetary orbits is such that at some epoch the two planets come so close to each other that their gravitational interaction dominates the attraction from the star. After that, the two planets are trapped in a close binary revolving around the sun. The gap in the lower right quarter in Fig. 11, at the collision area, is generated by this mechanism. For this reason the model we shall use in this study is the general three body problem, for planar motion, with the star as the more massive body and the two planets with small, but not negligible masses.

A very good tool to study the structure of the phase space is to find the position and the stability character of the periodic orbits or, equivalently, the fixed points of the Poincaré map on a surface of section. To make this clear, think of a two-dimensional Poincaré map on a surface of section. The position and the stability character of the fixed points (periodic orbits) play a crucial role on the structure of the phase space. A different position of the fixed points and/or a different stability character will change completely the topology of the phase space. This means that the fixed points/periodic orbits provide the framework of the phase space, although they are a set of measure zero.

Stable quasi periodic librations are expected to exist close to a stable periodic orbit. So, although the periodic orbits are a set of measure zero, they can be used as a tool to provide the regions of the phase space (or, equivalently, the space of orbital elements), where stable, quasi periodic librations exist. These stable librations are not periodic motion, but are close 
to an exact periodic motion. A real planetary system could exist at these regions. The periodic motion in whose vicinity we have stable librations is of two kinds: either non resonant, with small planetary eccentricities, or resonant, where the two planets are in a mean motion resonance. In this latter case the eccentricities are relatively large.

We remark that a periodic orbit is represented as a fixed point in the four dimensional phase space of the Poincaré map. A perturbed periodic orbit is defined by a four-dimensional deviation vector, and this makes the study of the properties of motion close to a periodic orbit quite complicated. For example, a possible perturbation could be to shift the planet $P_{2}$ on its orbit, or to rotate the orbit of $P_{2}$, at $t=0$, leaving all other elements the same. Or, we can change the value of the planetary eccentricities from those of the exact periodic motion, and/or the angle of apsides, leaving the semimajor axes the same, so that the resonance is not changed. Although a complete study along these lines is not available, we believe that several quasi periodic librations, as for example the cases studied by Nelson and Papaloizou (2002) are motions in the vicinity of exact periodic orbits.

It can be proved (Hadjidemetriou 1975) that families of periodic orbits in the planar general three body problem exist, in a rotating frame which will be defined in Sect. 2. This means that in a periodic orbit the relative configuration is repeated in phase space. In particular, in the planetary three body problem (one big body, the star and two small bodies, the planets) the periodic orbits are of two types:

- Non-resonant periodic orbits with nearly circular orbits of the two planets.

- Resonant periodic orbits with nearly elliptic orbits of the two planets.

The circular orbits are all symmetric but the elliptic orbits may be symmetric or asymmetric. Close to a stable periodic orbit there exists a region of stable librations, and it is at these regions that a planetary system could be trapped.

There exist families of elliptic periodic orbits for every mean motion resonance. As we will show, there are three topologically different resonant cases:

- The resonances of the form $(n+1): n,(2: 1,3: 2, \ldots)$.

- The resonances $(2 n+1):(2 n-1),(3: 1,5: 3, \ldots)$.

- All other resonances, $(5: 2,7: 3,8: 3, \ldots)$.

A global view of the resonant families of elliptic periodic orbits will be computed for each one of the above resonance types. There exist both symmetric and asymmetric families. As we shall see, the ratio of the planetary masses plays an important role on the stability and the existence of asymmetric families of periodic orbits. The sum of the masses of the planets also plays an important role on the stability and the existence of families of resonant periodic orbits. The stability of a symmetric periodic orbit depends, all other things being the same (semimajor axes, eccentricities), on the phase of the two planets, that is on whether the line of apsides are aligned or antialigned and on the position of the two planets at perihelion of aphelion at some epoch.

The properties of motion close to a periodic orbit are studied by considering a Poincaré map on a surface of section. Close to a stable periodic orbit we have stable librations and the motion in phase space takes place on a torus. On the contrary, close to an unstable periodic orbit we have irregular, chaotic, motion and in many cases the system disrupts into a binary system (the star and one planet) and an escaping planet.

The position of some real extrasolar planetary systems is compared with the above mentioned regions of stable librations. 


\section{The rotating frame}

The model that we will use is the general planar three body problem, for the star, which is the more massive body, and the two planets, with small, but not negligible masses. From this point on, the star will be called the sun and the planets will be denoted as $P_{1}$ and $P_{2}$. The indices 1 and 2 are for the inner and the outer planet, respectively, at least initially, because in a chaotic motion the roles of inner and outer planet may change. For intersecting planetary orbits, the index 1 will refer to the body with the smaller semimajor axis.

In the inertial frame where the center of mass of the system is at rest, we have four degrees of freedom. We define now a rotating frame of reference, whose origin is at the center of mass of the sun and the planet $P_{1}$, the $x$-axis is the line sun- $P_{1}$, the positive direction being from the sun to $P_{1}$, and the $y$-axis is perpendicular to the $x$-axis. As coordinates we use the position $x_{1}$ of $P_{1}$ on the $x$-axis, the coordinates $\left(x_{2}, y_{2}\right)$ of $P_{2}$ on the $x O y$ plane and the angle $\theta$ of the $x$-axis with a fixed direction in the inertial frame (Fig. 1). The $x O y$ frame is a non-uniformly rotating frame. The Lagrangian of the system in the above mentioned coordinates $x_{1}, x_{2}, y_{2}, \theta$ is

$$
\begin{aligned}
\mathcal{L}= & \frac{1}{2}\left(m_{1}+m_{0}\right)\left\{q\left(\dot{x}_{1}^{2}+x_{1}^{2} \dot{\theta}^{2}\right)+\frac{m_{2}}{m}\left[\dot{x}_{2}^{2}+y_{2}^{2}+\dot{\theta}^{2}\left(x_{2}^{2}+y_{2}^{2}\right)\right.\right. \\
& \left.\left.+2 \dot{\theta}\left(x_{2} \dot{y}_{2}-\dot{x}_{2} y_{2}\right)\right]\right\}-V,
\end{aligned}
$$

where

$$
V=-\frac{G m_{0} m_{1}}{r_{01}}-\frac{G m_{0} m_{2}}{r_{02}}-\frac{G m_{1} m_{2}}{r_{12}}
$$

and

$$
m=m_{0}+m_{1}+m_{2}, \quad q=m_{1} / m_{0} .
$$

$G$ is the gravitational constant and $r_{01}, r_{02}$ and $r_{12}$ are the distances between the sun and $P_{1}$, the sun and $P_{2}$ and $P_{1} P_{2}$, respectively. We note that the angle $\theta$ is ignorable, so besides the energy (or Jacobi) integral there also exists the angular momentum integral, $p_{\theta}=\partial \mathcal{L} / \partial \dot{\theta}=$ constant,

$$
p_{\theta}=\left(m_{0}+m_{1}\right)\left\{\dot{\theta}\left[q x_{1}^{2}+\frac{m_{2}}{m}\left(x_{2}^{2}+y_{2}^{2}\right)\right]+\frac{m_{2}}{m}\left(x_{2} \dot{y}_{2}-\dot{x}_{2} y_{2}\right)\right\} .
$$

By making use of the angular momentum integral, we can reduce the system to three degrees of freedom, in the variables $x_{1}, x_{2}$, and $y_{2}$. In this way, the study is restricted to the rotating frame $x O y$, which means that the relative motion of the three bodies in the rotating frame can be studied, independently of the motion of the system $x O y$ in the inertial frame. The Lagrangian of the reduced system in the rotating frame is the Routhian function $\mathcal{R}$,

$$
\begin{aligned}
\mathcal{R}= & \frac{1}{2}\left(m_{1}+m_{0}\right)\left\{q \dot{x}_{1}^{2}+\frac{m_{2}}{m}\left(\dot{x}_{2}^{2}+y_{2}^{2}\right)-\frac{\left[\frac{p_{\theta}}{m_{1}+m_{2}}-\frac{m_{2}}{m}\left(x_{2} \dot{y}_{2}-\dot{x}_{2} y_{2}\right)\right]^{2}}{q \dot{x}_{1}^{2}+\frac{m_{2}}{m}\left(x_{2}^{2}+y_{2}^{2}\right)}\right\}, \\
& -V .
\end{aligned}
$$

Note that the angular momentum $p_{\theta}$ appears as a fixed parameter.

From this point on, we shall restrict our study to the rotating frame only. In order to avoid duplication of the results, we must fix the units of mass, length and time, by considering three 
Fig. 1 The rotating frame $x O y$. The planet $P_{1}$ moves on the $x$-axis and the planet $P_{2}$ moves in the $x O y$ plane. The angle $\theta$ defines the orientation of the rotating frame

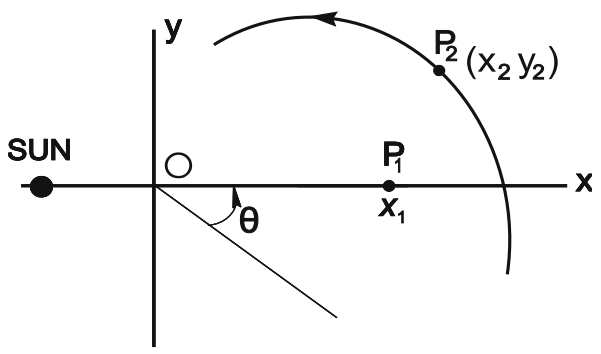

normalizing conditions:

$$
m_{0}+m_{1}+m_{2}=1, \quad G=1, \quad p_{\theta}: \text { fixed. }
$$

This means that the unit of mass is the total mass of the system, the gravitational constant is taken equal to unity and the constant angular momentum is given a fixed value (note that the angular momentum appears as a fixed parameter in the differential equations in the rotating frame).

It can be proved (Hadjidemetriou 1975) that families of periodic orbits exist in the rotating frame $x O y$. In particular, we are interested in periodic orbits of the planetary type, that we shall study in the next section.

\section{Periodic orbits of the planetary type}

We consider a three-body system with the sun $S$ as the main body and two small, but gravitationally interacting, planets $P_{1}$ and $P_{2}$. As we mentioned above, families of periodic orbits exist in the rotating frame $x O y$. A periodic orbit can be symmetric or asymmetric with respect to the rotating $x$-axis. We remind that in all cases $P_{1}$ moves on the $x$-axis and $P_{2}$ in the $x O y$ plane. In a symmetric periodic orbit, for a certain epoch, for example $t=0$, it is $\dot{x}_{1}=0$ and $\dot{x}_{2}=0$. This implies that at $t=0$ the planet $P_{2}$ crosses perpendicularly the $x$-axis and at that time the planet $P_{1}$ is temporarily at rest on the $x$-axis. So, the non zero initial conditions of a periodic orbit are: $x_{10}, x_{20}, \dot{y}_{20}$, which means that a family of symmetric periodic orbits can be represented by a one-dimensional curve in the three-dimensional space of initial conditions $x_{10}, x_{20}, \dot{y}_{20}$. The orbits for which we cannot find an epoch for which the above conditions are satisfied, are asymmetric.

There are two different types of periodic orbits of the planetary type:

- Periodic orbits with the two planets describing nearly circular orbits. In general, the system is non resonant. This is seen in Fig. 2. We remark that the planetary orbits are exactly circular in the unperturbed case, and nearly circular if the gravitational interaction between the planets is introduced. All these orbits are symmetric with respect to the $x$-axis of the rotating frame.

- Periodic orbits with the two planets describing nearly elliptic orbits. In the unperturbed case the two planetary orbits are exactly elliptic. We remark that, contrary to the circular case, all elliptic periodic orbits are resonant, corresponding to a mean motion resonance. We can distinguish between symmetric and asymmetric resonant elliptic periodic orbits.

- Symmetric elliptic periodic orbits: the two planets describe elliptic orbits whose lines of apsides are aligned or antialigned, and in addition, when $P_{1}$ is at perihelion or aphelion, $P_{2}$ is at that time also at perihelion or aphelion (Fig. 3a). This means that there 
Fig. 2 The circular motion of the two planets $P_{1}$ and $P_{2}$. The motion is periodic in the rotating frame $x O y$, for any value of the planetary semimajor axes
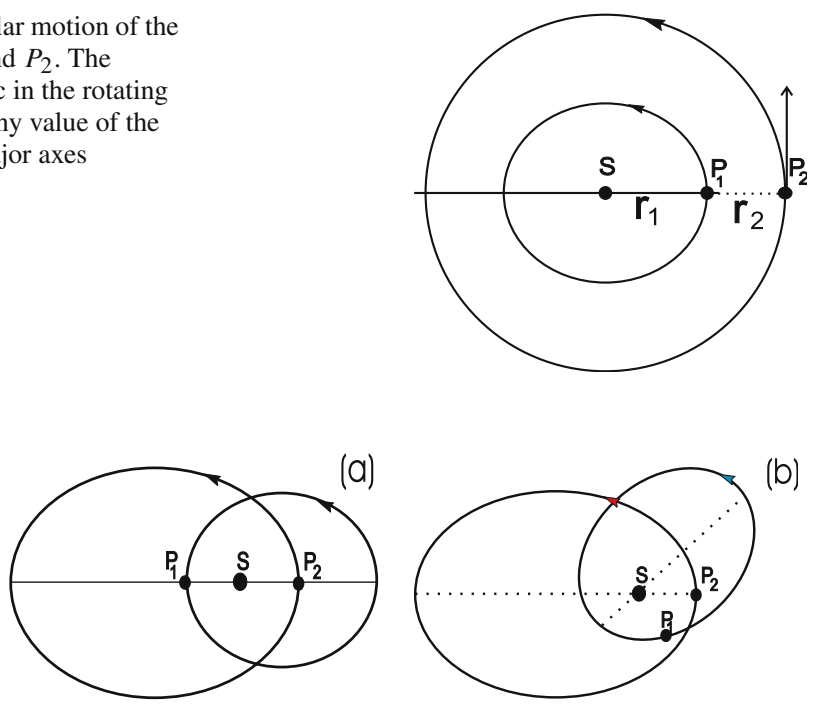

Fig. 3 (a) The elliptic motion of the two planets $P_{1}$ and $P_{2}$ in the symmetric configuration. (b) The elliptic motion of the two planets in the asymmetric configuration

are four different initial configurations, which are equivalent in pairs, i.e. position at $t=0$ and $t=T / 2, T$ being the period, depending on the particular resonance.

- Asymmetric elliptic periodic orbits: The lines of apsides of the two planets are not in the same line (Fig. 3b).

\section{Families of periodic orbits}

It can be proved that families of periodic orbits of the planetary type exist, in a rotating frame, in the general three-body problem (Hadjidemetriou 1976). In order to obtain a global view of the resonant and non resonant periodic orbits, we will start with the unperturbed problem (zero planetary masses) and circular planetary orbits, and study how things change when we give masses to the planets.

\subsection{The unperturbed family of circular orbits}

Consider the two planets $P_{1}$ and $P_{2}$ describing circular orbits in the plane (Fig. 2). It is evident that the motion is periodic in the rotating frame $x O y$ defined in Sect. 1 , for all values of the semimajor axes (radii) of the two planets. The planet $P_{1}$ moves on the $x$-axis (in this particular case it is fixed) and the planet $P_{2}$ moves in the $x O y$ plane. This motion is symmetric with respect to the $x$-axis, because for some time, say $t=0$, it is $\dot{x}_{1}=0$ (this is always the case) and at that time the planet $P_{2}$ crosses perpendicularly the $x$-axis, $\dot{x}_{2}=0$. These periodic orbits belong to a monoparametric family of symmetric periodic orbits. To make this clear, let us fix the value of the semimajor axis $a_{1}$ of $P_{1}$ and vary the semimajor axis $a_{2}$ of $P_{2}$. In this way, we obtain a continuous family of periodic orbits, along which the ratio $a_{1} / a_{2}$ of the semimajor axes, or, equivalently, the ratio $n_{1} / n_{2}$ of the frequencies varies. Evidently $n_{1} / n_{2}$ passes through rational values, which means that on this single family of periodic orbits there exist resonant orbits, corresponding to mean motion resonance between the planets. 
Fig. 4 The unperturbed family of circular orbits (lower curve) with three representative resonances, 2:1, 3:1, and 5:2 (schematically). The upper curves represent the evolution of the single circular family, when the gravitational interaction between the planets is introduced. A gap appears at the 2:1 and two families of 2:1 resonant elliptic orbits bifurcate from the gap

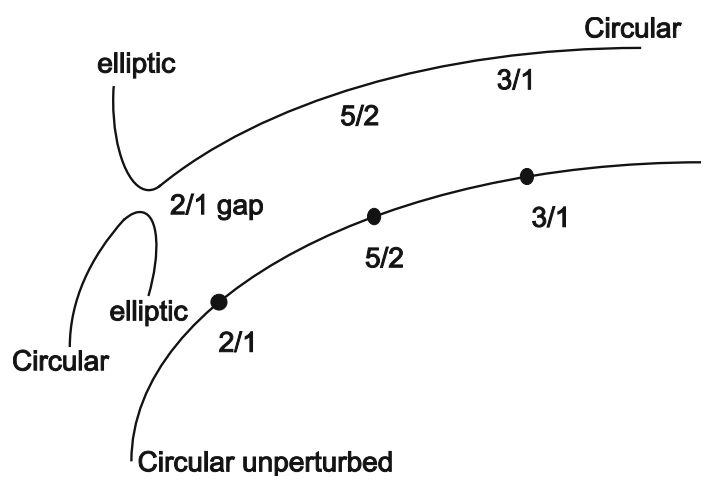

\subsection{Continuation of the circular family to non zero planetary masses}

We give now small, but not negligible masses to the two planets. It can be proved (Hadjidemetriou 1976) that all the unperturbed circular periodic orbits are continued, with the same period, as periodic orbits with nearly circular planetary orbits, in the rotating frame $x O y$. These orbits are symmetric with respect to the $x$-axis. The continuation is not possible only at the resonances of the form $(n+1): n$, i.e, $2: 1,3: 2,4: 3, \ldots$ At these resonances a gap appears, as we show schematically in Fig. 4, using the 2:1 resonance as a representative example. At such a gap, we have a bifurcation of two families of 2:1 resonant elliptic periodic orbits, as we shall see in the following.

\subsection{Evolution of the stability on the family of circular orbits}

It is obvious that all the orbits of the circular unperturbed family are orbitally stable, because the two planetary orbits are uncoupled Keplerian orbits. We have, in the rotating frame, three degrees of freedom and consequently, we have three pairs of unit eigenvalues, which are all on the unit circle in the complex plane (for the theory see Hadjidemetriou 2006). One pair of eigenvalues is equal to one, because of the existence of the energy integral. The other two pairs of eigenvalues are complex conjugate on the unit circle and are obtained, by Keplerian theory, to be equal to

$$
\lambda_{1,2}=e^{ \pm i \phi}, \quad \lambda_{3,4}=e^{ \pm i \phi}, \quad \phi=\frac{2 \pi}{1-n_{2} / n_{1}},
$$

where $n_{1}, n_{2}$ are the frequencies of the two planets in their orbits (in the inertial frame). We see that $\lambda_{1}=\lambda_{3}$ and $\lambda_{2}=\lambda_{4}$, i.e. there is a double eigenvalue equal to $e^{i \phi}$ and a double complex conjugate eigenvalue equal to $e^{-i \phi}$ (Fig. 5a).

As we mentioned in the previous section, the ratio $n_{1} / n_{2}$ varies along the family of periodic orbits of the unperturbed problem and consequently passes through rational values, which correspond to mean motion resonance between the two planets. This means that the double eigenvalue $\lambda_{1}=\lambda_{3}$ (and its complex conjugate) move on the unit circle, as we move on the unperturbed family of circular periodic orbits. From Eq. 6 , we see that the ratio $\phi / 2 \pi$ takes rational values at all resonant orbits on the family, and, in general, the double eigenvalue $\lambda_{1}=\lambda_{3}$ is not at +1 or -1 .

Let us now switch on the planetary masses, introducing the gravitational interaction between the two planets. The double eigenvalue $\lambda_{1}=\lambda_{3}$ (and its complex conjugate) split into two separate eigenvalues and move in the complex plane, but must obey the Hamiltonian 


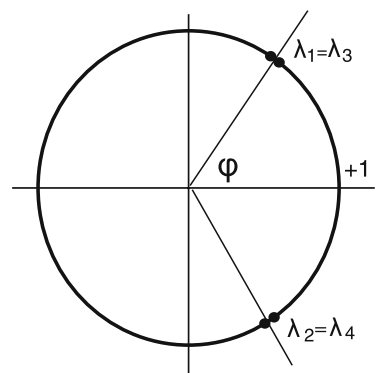

(a)

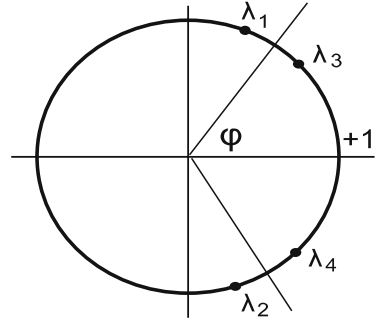

(b)

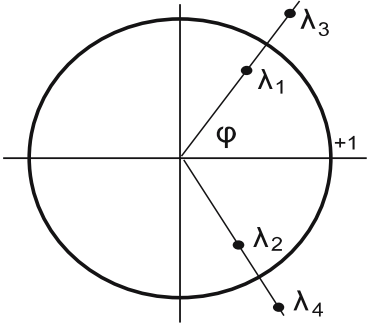

(c)

Fig. 5 (a) The two double pairs of eigenvalues $\lambda_{1}=\lambda_{3}$ and $\lambda_{2}=\lambda_{4}$ of the unperturbed problem on the unit circle in the complex plane. (b) The two double eigenvalues split and remain on the unit circle when the gravitational interaction of the two planets is taken into account. The stability is preserved. (c) The evolution of the four eigenvalues outside the unit circle, generating complex instability. This cannot happen in a planetary system

properties: they must be arranged in reciprocal pairs and also in complex conjugate pairs. This means that we have two possibilities: either to move on the unit circle, as shown in Fig. 5b, preserving the stability, or move outside the unit circle, as shown in Fig. 5c (complex conjugate pairs: $\left(\lambda_{1}, \lambda_{2}\right),\left(\lambda_{3}, \lambda_{4}\right)$, reciprocal pairs $\left.\left(\lambda_{1}, \lambda_{4}\right),\left(\lambda_{2}, \lambda_{3}\right)\right)$, generating complex instability. It can be proved however, by making use of the theory of Krein (Hadjidemetriou 1982), that the evolution to complex instability, when $\phi \neq 0$ or $\pi$ (the eigenvalues are not at \pm 1 ), is impossible in a planetary system with two planets moving in the plane in the same direction. In fact the proof is stronger. It is proved that there does not exist a Hamiltonian perturbation to generate complex instability in such a system.

The only case where the perturbation can generate instability is when two eigenvalues meet at +1 or at -1 , on the unit circle in the complex plane, as the corresponding orbit moves on the family of circular orbits. The first case, meeting of the eigenvalues at +1 , corresponds to a resonance of the form $(n+1): n$, i.e., $2: 1,3: 2, \ldots$ and the second case, meeting of the eigenvalues at -1 , corresponds to a resonance of the form $(2 n+1):(2 n-1)$, i.e., $3: 1$, $5: 2, \ldots$ But in the first case the continuation is not possible (see the gap in Fig. 4), so we will consider the case for the resonances $3: 1,5: 3, \ldots$

Let us consider the region on the perturbed circular family close to the $3: 1$ resonance. The two double pairs of complex conjugate eigenvalues $\lambda_{1}=\lambda_{3}$ and $\lambda_{2}=\lambda_{4}$ have split on the unit circle to two separate complex conjugate pairs $\left(\lambda_{1}, \lambda_{2}\right)$ and $\left(\lambda_{3}, \lambda_{4}\right)$ (Fig. 5b) and each pair moves toward the point -1 . When they meet at -1 , the eigenvalues may move outside the unit circle, on the real axis, and thus generate instability, as predicted by the theory of Krein (Hadjidemetriou 1982). This is seen in Fig. 6. It may happen that only one pair of eigenvalues, $\left(\lambda_{1}, \lambda_{2}\right)$, goes outside the unit circle, and the other, $\left(\lambda_{3}, \lambda_{4}\right)$, staying on the unit circle, or both pairs of eigenvalues go outside the unit circle. This depends on the magnitude of the masses, as we shall see in the following. After we cross the 3:1 resonance, the eigenvalues return again on the unit circle, restoring the stability.

From the above, we see that the only region on the family of circular periodic orbits which is unstable is the region close to resonances of the form 3:1, 5:3,...All other resonant and non-resonant cases are stable.

\subsection{Families of symmetric and asymmetric elliptic periodic orbits}

The periodic motion of a planetary system with elliptic orbits of the two planets is necessarily resonant, with the two planets locked in a mean motion resonance. All these orbits belong 
Fig. 6 (a) The position of the complex conjugate eigenvalues $\lambda_{1}, \lambda_{2}$ on the unit circle close to the 3:1 resonance. (b) The evolution of the eigenvalues $\lambda_{1}, \lambda_{2}$ outside the unit circle when the orbit crosses the $3: 1$ resonance
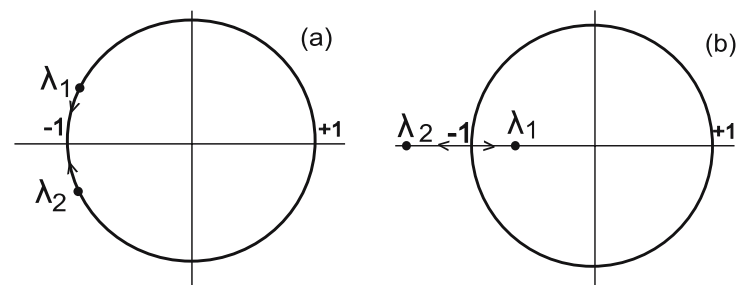

to monoparametric families, along which the eccentricities of the two planetary orbits vary, and, in the asymmetric case, the difference $\Delta \varpi$ between the angle of apsides also varies. In order to obtain a global view of the families of elliptic periodic orbits, we will start with the families that bifurcate from the family of circular periodic orbits. Next, we shall study the elliptic families that bifurcate from the above mentioned elliptic families. We remark however that apart from these two categories of elliptic families, there also exist families of elliptic orbits that appear as independent from them. Some of these latter families may be the continuation of families of the elliptic restricted three body problem, by giving mass to the massless body.

As we will show in the following, the orbits of the circular family are all symmetric with respect to the $x$-axis of the rotating frame. The families of elliptic orbits that bifurcate from the circular family are also symmetric. However, the orbits of the elliptic families that bifurcate from the elliptic families are all asymmetric. The independent elliptic families are either symmetric or asymmetric.

Consider a family of periodic orbits, either circular or elliptic. A bifurcation of a new family of periodic orbits is only possible from that orbit on the family which has an eigenvalue equal to +1 (Hadjidemetriou 2006). We remark that this is a double unit eigenvalue, because the eigenvalues are always in complex conjugate pairs. We call this orbit a critical orbit. This critical orbit may be the point on the family where we have a change from stability to instability (or vice versa), but it can also be, on the circular family, any resonant $p: q$ periodic orbit, if it is described several times, as we show in the following.

We will consider separately the bifurcations from the circular family and from the elliptic families.

\subsubsection{Bifurcation from the circular family}

We shall show now that from each resonant region on the family of circular periodic orbits we have a bifurcation of families of elliptic periodic orbits. There are two elliptic families bifurcating from a $p: q$ resonant periodic orbit if $p: q \neq 3: 1,5: 3, \ldots$ and two, or four families if $p: q=3: 1,5: 3, \ldots$, as we shall prove below. All these families are symmetric with respect to the rotating $x$-axis.

As we mentioned in Sect. 4.3, there are three pairs of eigenvalues: one unit pair, due to the existence of the energy integral and two more complex conjugate pairs, on the unit circle. In the unperturbed case (zero planetary masses) these two complex conjugate pairs coincide,

$$
\lambda_{1}=\lambda_{3}=e^{+i \phi}, \quad \lambda_{2}=\lambda_{4}=e^{-i \phi}, \quad \phi=\frac{2 \pi}{1-n_{2} / n_{1}},
$$

where $n_{1} / n_{2}$ is the ratio of the mean motions of the two planets. This ratio is not, in general, rational. It is at the rational (resonant) points that a bifurcation of families of elliptic periodic orbits is possible. In addition, a bifurcation of a family of elliptic orbits is possible at all 
(a)

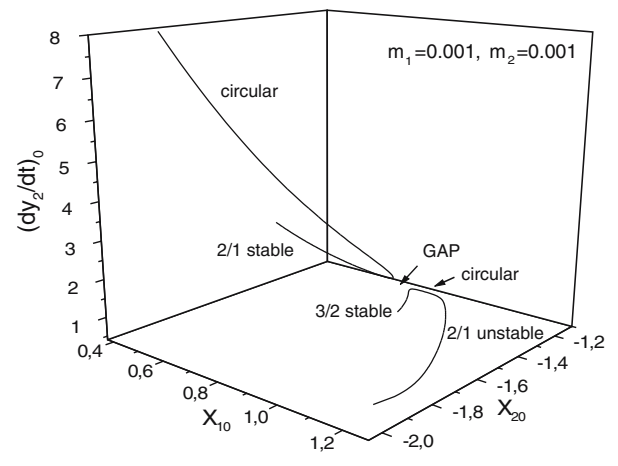

(b)

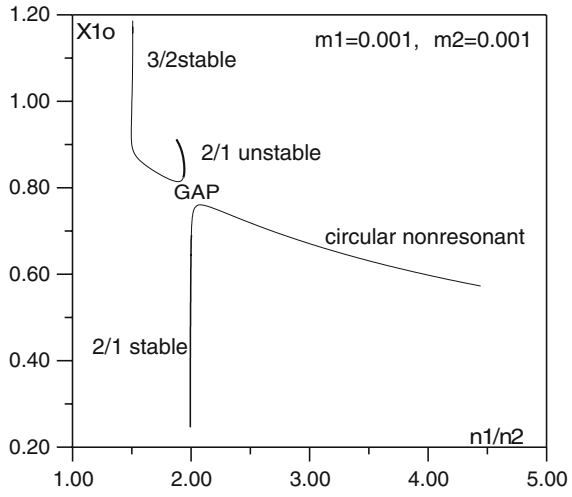

Fig. 7 (a) The circular and the elliptic families of periodic orbits at the 2:1 resonance, for a fictitious system with $m_{0}=0.998, m_{1}=0.001$, and $m_{2}=0.001$. The gap is clearly seen. (b) The same system in the space $\left(n_{1}: n_{2}\right)-x_{10}$ The $3: 2$ resonant family is also shown

points on the circular family where we have a change from stability to instability, or vice versa. This latter case happens at the resonances of the form $3: 1,5: 3, \ldots$ In this case, we may have a bifurcation of two or of four families of resonant elliptic periodic orbits.

There are three topologically different resonances:

- The resonances of the form $n_{1} / n_{2}=(n+1) / n, n=1,2, \ldots$

These are the resonances $2: 1,3: 2, \ldots$ At these resonances, the periodic orbits are not continued as circular periodic orbits, but instead, a gap appears on the continued circular family and at both ends of the gap we have the beginning of a family of resonant elliptic periodic orbits, corresponding to the $(n+1): n$ resonance (Fig. 3). A typical example is shown in Fig. 7, for a planetary system with masses $m_{0}=0.998, m_{1}=0.001$, and $m_{2}=0.001$. In Fig. 7a, we show the circular family of symmetric periodic orbits, in the space of initial conditions $x_{10} x_{20} \dot{y}_{20}$, the gap at the 2:1 resonance and the bifurcation from the gap of two families of elliptic symmetric periodic orbits. In Fig. 7b, we show the same diagram in the space $\left(n_{1}: n_{2}\right)-x_{10}$. The gap at the 2:1 resonance and the elliptic resonant periodic orbits are clearly seen. In Fig. $7 b$ the $3: 2$ resonant elliptic family is also shown.

- The resonances of the form $n_{1}: n_{2}=(2 n+1):(2 n-1), n=1,2, \ldots$

These are the resonances $3: 1,5: 3, \ldots$ As we mentioned in Sect. 4.3, instability is generated in a small region close to the above resonances. As shown schematically in Fig. 6, only one pair of eigenvalues may go outside the unit circle, generating simple instability, or both pairs may go outside the unit circle, generating double instability. Whether we have the first or the second case, it depends on the magnitude of the perturbation, i.e. on the total mass of the planets. This is shown in Fig. 8, where we plot the stability index along the family of circular orbits, close to the 3:1 resonance, for two different planetary masses. The stability index corresponds to a complex conjugate pair of eigenvalues and it is defined as

$$
b_{1}=\lambda_{1}+\lambda_{2}, \quad b_{2}=\lambda_{3}+\lambda_{4} .
$$

This means that we have stability if

$$
\left|b_{i}\right| \leq 2 \quad(i=1,2) .
$$



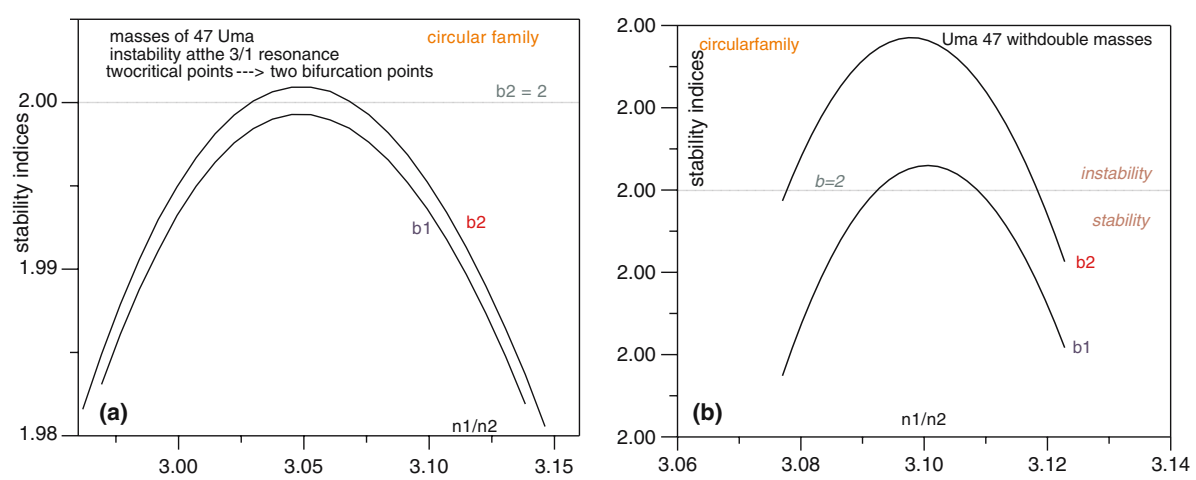

Fig. 8 (a) The stability index along the circular family, close to the $3: 1$ resonance, in the space $n_{1}: n_{2}$ stability indices for the masses of the system $47 \mathrm{UMa}, m_{0}=0.998, m_{1}=0.001$, and $m_{2}=0.001$. Two critical points exist. (b) The same as in (a), but with double planetary masses. Four critical points exist now

In Fig. 8a, we use the (normalized) masses of the system 47 UMa, $m_{0}=0.996942, m_{1}=$ 0.002354 , and $m_{2}=0.000704$, (Fisher et al. 2002) as an example and in Fig. $8 \mathrm{~b}$ the double masses. In the first case only one stability index becomes unstable for a small region close to the 3:1 resonance, but as the perturbation (the masses) increases, both stability indices become unstable. At the points where the stability changes, the stability index is equal to +2 , which means that the eigenvalues are equal to $e^{ \pm i \pi}=-1$. If now this orbit is described twice, the eigenvalue becomes equal to $\left(e^{ \pm i \pi}\right)^{2}=+1$, and consequently the orbit is critical and a bifurcation of a family of elliptic periodic orbits is possible. The period of the elliptic orbits is equal to twice the period of the circular orbit from which it bifurcates.

In the former case we have two critical points, and consequently two elliptic 3:1 resonant periodic orbits. In the latter case there are four critical points and consequently four families of resonant 3:1 elliptic periodic orbits. All these families are symmetric with respect to the rotating $x$-axis and differ in the initial phase only, i.e. on whether the lines of apsides are aligned or antialigned and on the position of the two planets at perihelion or at aphelion at $t=0$. Along these families the planetary eccentricities vary, starting with zero values.

- All other resonances $n_{1}: n_{2} \neq(n+1): n,(2 n+1):(2 n-1), n=1,2, \ldots$

These are resonances of the form 5:2, 7:3, 5:1, 8:3, .. As we mentioned in Sect. 4.3, the stability is preserved when we go from the perturbed to the unperturbed problem, which means that the eigenvalues $\lambda_{1}=\lambda_{3}$ and $\lambda_{2}=\lambda_{4}$ split, but stay on the unit circle. Let us assume now that we approach a $p / q$ resonance $\left(n_{1} / n_{2}=p / q\right.$, $p / q \neq(n+1) / n,(2 n+1) /(2 n-1))$ on the perturbed family. The eigenvalues $\lambda_{1}, \lambda_{3}$ move on the unit circle and cross the point

$$
e^{i 2 \pi \frac{q}{q-p}}
$$

one after the other. So, there are two orbits on the perturbed family of periodic orbits, close to each other, and close to the $p: q$ resonance, which have a pair of eigenvalues $\lambda_{1}, \lambda_{3}$ (or $\lambda_{2}, \lambda_{4}$ ) equal exactly to

$$
e^{ \pm i 2 \pi \frac{q}{q-p}}
$$

This is not a critical orbit (it does not have a unit pair of eigenvalues). However, it can become critical if this orbit is described $|q-p|$ times, because its eigenvalues are equal to

$$
\lambda_{i}^{|q-p|}=e^{ \pm i 2 \pi q}=+1 .
$$




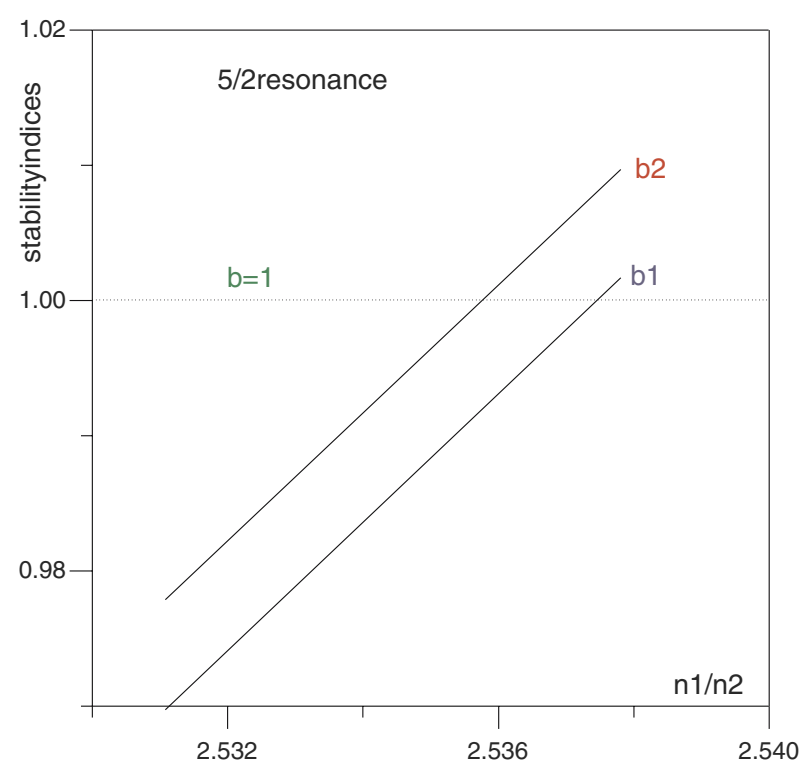

Fig. 9 The two stability indices close to the 5:2 resonance along the perturbed family of circular orbits. They cross the value $b=1$, corresponding to the pair of eigenvalues $e^{ \pm i 2 \pi / 3}$

At this point we have a bifurcation of a $p: q$ family of resonant elliptic periodic orbits, along which the planetary eccentricities vary, starting from zero values. The period along this resonant family is $|p-q|$ times the period of the corresponding circular resonant periodic orbit. Since there are two such points, there are two $p: q$ resonant families of elliptic periodic orbits that bifurcate from the circular family at the region close to the $p: q$ resonance. As an example, we consider the region close to the 5:2 resonance on the family of circular periodic orbits. There exist two distinct periodic orbits, close to each other, with eigenvalues $\lambda_{1,2}=e^{ \pm 2 i \pi / 3}$ and $\lambda_{3,4}=e^{ \pm 2 i \pi / 3}$, respectively (stability indices $b_{1}=+1$, $b_{2}=+1$, respectively). From each one there is a bifurcation of a 5:2 resonant elliptic symmetric family of periodic orbits, with period three times the period of the circular orbit from which they bifurcate. This is shown in Fig. 9, for the masses of $47 \mathrm{UMa}$, used also in the 3:1 resonance above. These orbits differ in the phase only and the planetary eccentricities vary, starting with zero values.

\subsubsection{Bifurcation from the elliptic families}

As we mentioned before, there is a bifurcation of a family of periodic orbits from each orbit of a family which is critical, i.e. it has a pair of eigenvalues equal to +1 . This is the case where we have a change of stability to instability, or vice versa, as we have already seen in the 3:1 resonance case on the family of circular orbits. Let us consider now a family of elliptic periodic orbits that bifurcates from the circular family. It may happen that the stability along the family changes. At this point there is a bifurcation of a new family of elliptic periodic orbits. The numerical computations have shown that all such elliptic families are asymmetric. We remark that in the unperturbed case the planetary orbits of an elliptic family are uncoupled elliptic Keplerian orbits in resonance, which implies that there are two pairs of eigenvalues 

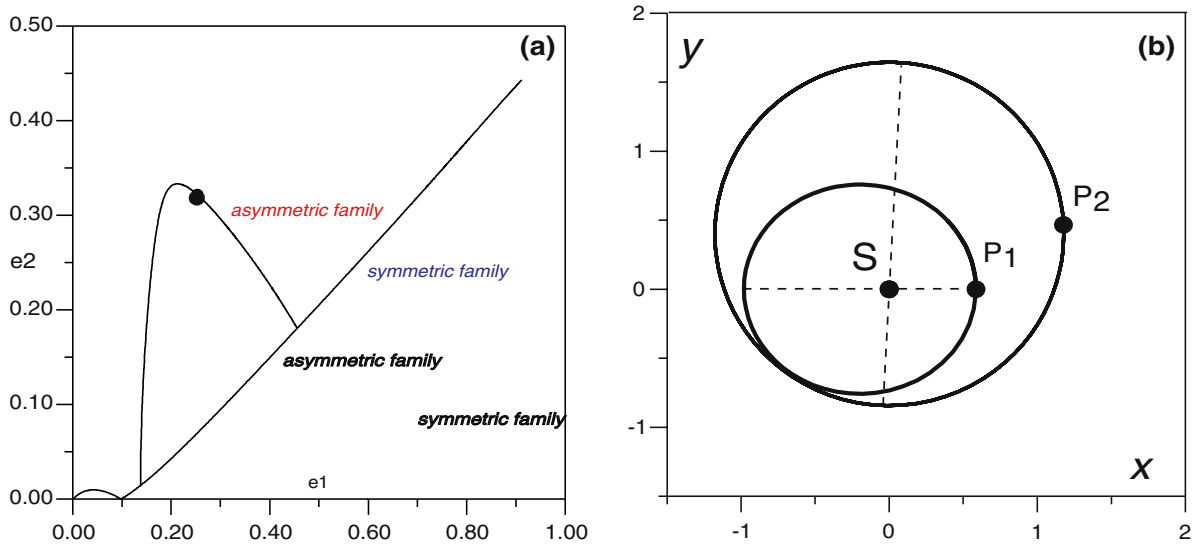

Fig. 10 (a) The family of symmetric elliptic periodic orbits, in the eccentricity space, for the masses of the Io-Europa satellites of Jupiter, and the bifurcation of an asymmetric family starting from one end of the unstable region and ending to the other end of the unstable region. (b) The asymmetric orbit indicated on the asymmetric family of (a)

equal to +1 (in addition to the third unit pair which always exists, due to the existence of the energy integral). When the perturbation is applied one, or both, of these unit pairs may go outside the unit circle in the complex plane, on the real axis, and thus generate instability. This depends mainly on the ratio $m_{1} / m_{2}$ of the planetary masses, as we shall show by some examples that follow.

An example of bifurcation of an asymmetric family of elliptic periodic orbits, for the masses of the Io-Europa satellites of Jupiter at the 2:1 resonance, is given in Fig. 10a. The normalized masses are $m_{0}=0.999928, m_{1}=0.000047$, and $m_{2}=0.000025$. Note that $m_{1}>m_{2}$. On the symmetric family there is an unstable region and from both ends of it, at the critical points, there is a bifurcation of an elliptic 2:1 resonant asymmetric family of periodic orbits. It turns out that these two families coincide, as shown in Fig. 10a. In Fig. 10b, we present a representative asymmetric orbit, corresponding to the one indicated in Fig. 10a. We remark that the unstable region on the symmetric elliptic family appears because it is $m_{1}>m_{2}$. If it were $m_{1}<m_{2}$, the whole symmetric family is stable and consequently there are no critical points from which to bifurcate a new family of elliptic periodic orbits. The critical mass ratio, beyond which an unstable region appears is $m_{1} / m_{2}=0.97$ (Ferraz-Mello et al. 2003).

Finally we remark, that apart from all the above mentioned, symmetric or asymmetric families of resonant elliptic periodic orbits, there exist also such families that do not bifurcate from the above mentioned families. Some of them are the continuation from the elliptic restricted problem to the general problem, if we give mass to the massless body.

\section{Factors that affect the stability of a planetary system}

Families of periodic orbits for the masses of some observed extrasolar planetary systems were studied in several papers: Hadjidemetriou (2002), Hadjidemetriou and Psychoyos (2003), Psychoyos and Hadjidemetriou (2005), and Voyatzis and Hadjidemetriou (2005, 2006), studying symmetric and asymmetric periodic orbits for the systems Gliese 876, HD 82943, 
Fig. 11 The three families of symmetric periodic orbits at the 2:1 resonance in the eccentricity space, for the masses of HD 82943 given by Israelian et al. (2001). A positive eccentricity denotes position at aphelion and a negative eccentricity position at perihelion. The positions of HD 82943 , for the four different initial configurations are also shown

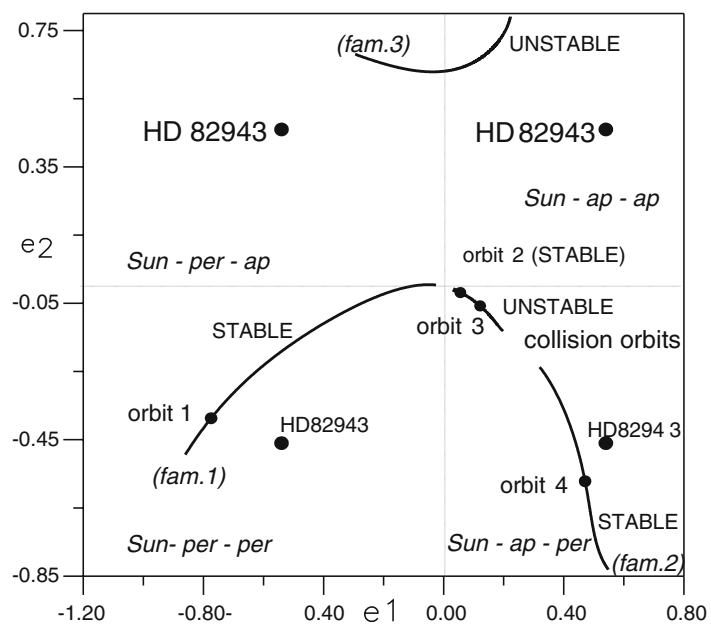

at the $2: 1$ resonance, $55 \mathrm{CnC}$ at the $3: 1$ resonance, $47 \mathrm{UMa}$ at the 5:2 resonance and also for the Io-Europa satellites of Jupiter at the 2:1 resonance. Stationary solutions close to mean motion resonance, which in fact correspond to periodic orbits, have been studied by Beauge et al. (2003, 2006). It is not the object of the present paper to review these results, but to use some of them as examples to make clear the factors that affect the stability of a planetary system and to show the stability regions close to a linearly stable periodic orbit, by making use of the Poincare map on a surface of section. In particular, we will start with the families of periodic orbits corresponding to the masses of the system HD 82943. Although the elements of this system have been revised several times, the fit given by Israelian et al. (2001), that we shall use here, is enough for our purpose. The elements of this fit are: $m_{0}=1.05 M_{\mathrm{SUN}}$, $m_{1} \sin i=0.88 \mathrm{MJ}, m_{2} \sin i=1.63 \mathrm{MJ}, a_{1}=0.73 \mathrm{AU}, a_{2}=1.16 \mathrm{AU}, e_{1}=0.54 \pm 0.05$, and $e_{2}=0.41 \pm 0.08$. In Fig. 11, we present three families of symmetric periodic orbits, for the normalized masses corresponding to the above system, in the space $e_{1} e_{2}$, using as a convention $e_{i}>0$ to denote position at aphelion and $e_{i}<0$ position at perihelion. There is a stable family, family 1 where the line of apsides are aligned and the two planets are both at perihelion, at $t=0$, and another family, family 2, which starts as stable and becomes unstable at a region where close encounters between the two planets take place. We remark that the whole family 1 is stable because $m_{1}<m_{2}$. After this collision region, at relatively large eccentricities, the family is again stable. Along this family, the lines of apsides are antialigned and at $t=0 P_{1}$ is at aphelion and $P_{2}$ at perihelion. These two families bifurcate from the circular family at the region where the 2:1 resonant gap appears. A third family, family 3, independent from these two also exists, and is unstable. In this diagram, we also show the position of the system HD 82943, with its elements as given above, for all four initial different configurations and we shall comment on their stability in the next sections.

The behavior of the system when a periodic orbit is perturbed, is studied by the Poincare map on a surface of section. As we shall see in the following, stable librations exist close to a stable periodic orbit and chaotic motion appears for larger deviations from the exact periodic motion of a stable orbit or for perturbations close to an unstable periodic orbit. The Poincaré map is defined on the surface of section (Fig. 12)

$$
H=h=\text { constant }, \quad y_{2}=0, \quad \dot{y}_{2}>0
$$


Fig. 12 The Poincaré map on a surface of section, defined at the times when $P_{2}$ crosses the $x$-axis in the positive sense

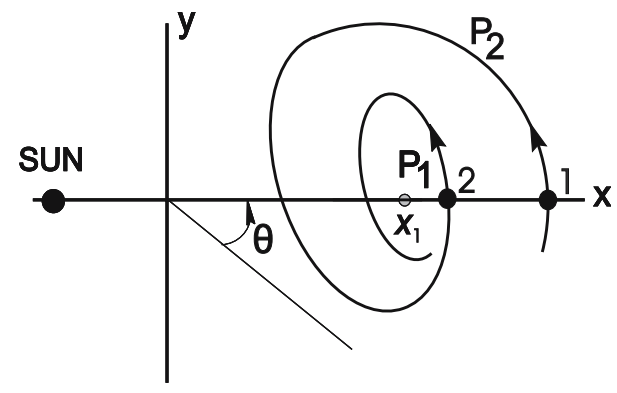

and is in the four-dimensional space $x_{1}, \dot{x}_{1}, x_{2}$, and $\dot{x}_{2}$. In some examples that we shall show below, we give the projections in some coordinate plane of the phase space of the Poincaré map.

\subsection{Deviation from the symmetry of a stable periodic orbit: region of stability}

In Fig. 13, we consider a typical orbit on the family 1, orbit 1, and study its stability, by shifting the position of $P_{2}$ on its orbit at $t=0$, thus destroying the symmetry. This orbit corresponds to the configuration where the line of apsides are aligned and both planets are at perihelion, at $t=0$. We note that for a range of deviations from the exact periodic motion, the perturbed motion takes place on a well defined torus. Although the phase space of the Poincaré map is four dimensional and consequently the deviations from the periodic motion that we consider do not cover all possible directions, it is clear that close to a stable periodic orbit there exists a region of stable librations, with small amplitude, close to the 2:1 resonance.

The same is repeated for a typical orbit on the family 2, orbit 4, lying on the stable part beyond the collision area (Fig. 14). The planetary orbits are antialigned and $P_{1}$ lies at aphelion while $P_{2}$ at perihelion, at $t=0$. We note that although the eccentricities are large and the planetary orbits intersect, there exists a region of stable librations.

\subsection{Comparison between a linearly stable and an unstable orbit}

We shall study here the importance of the linear stability or instability of a periodic orbit, with respect to the properties of the phase space close to such an orbit. As examples we shall use two periodic orbits on the family 2, with small eccentricities, one stable and the other unstable. As in the previous case, we perturb the system by destroying the symmetry by shifting the planet $P_{2}$ on its orbit. Close to the stable orbit (Fig. 15) we have stable librations, and the motion takes place on a well defined torus. On the contrary, close to the unstable periodic orbit (Fig. 16), the motion is finally chaotic. It is worth noting that for a relatively long time interval the motion looks as a stable libration, on a torus, but later it becomes chaotic.

\subsection{Dependence of the stability on the phase}

We consider here the motion corresponding to the four different configurations of the extrasolar system HD 82943 (old fit mentioned above), for the four different phases shown in Fig. 11. The behavior is shown in Fig. 17. Note that the configurations: (alignment, $P_{1}, P_{2}$ at perihelion) and (antialignment, $P_{1}$ at aphelion, $P_{2}$ at perihelion) are stable, while the other two configurations, (alignment, $P_{1}, P_{2}$ at aphelion) and (antialignment, $P_{1}$ at perihelion, $P_{2}$ 

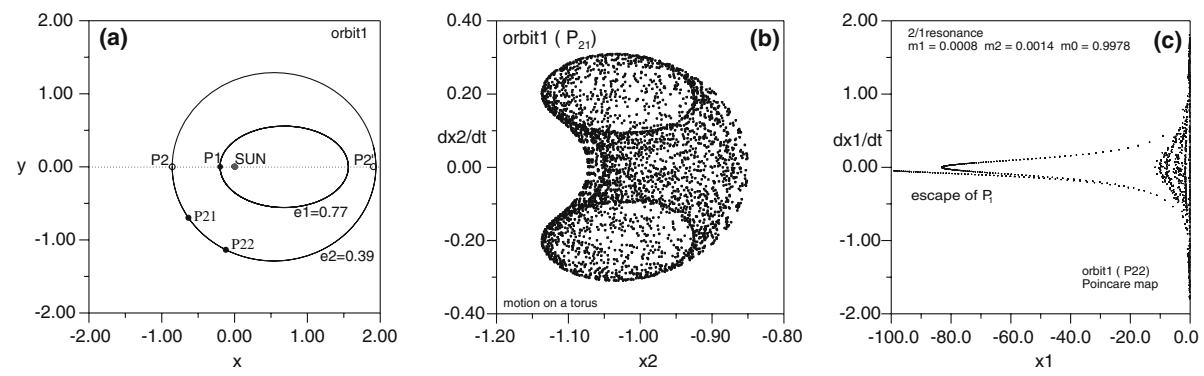

Fig. 13 (a) The stable orbit 1 and the two shifted positions, $P_{21}$ and $P_{22}$ of $P_{2}$ on its orbit. (b) The Poincaré map: $P_{2}$ at $P_{21}$, resulting to stable librations. (c) The Poincaré map: $P_{2}$ at $P_{22}$, resulting to chaotic motion and disruption of the system
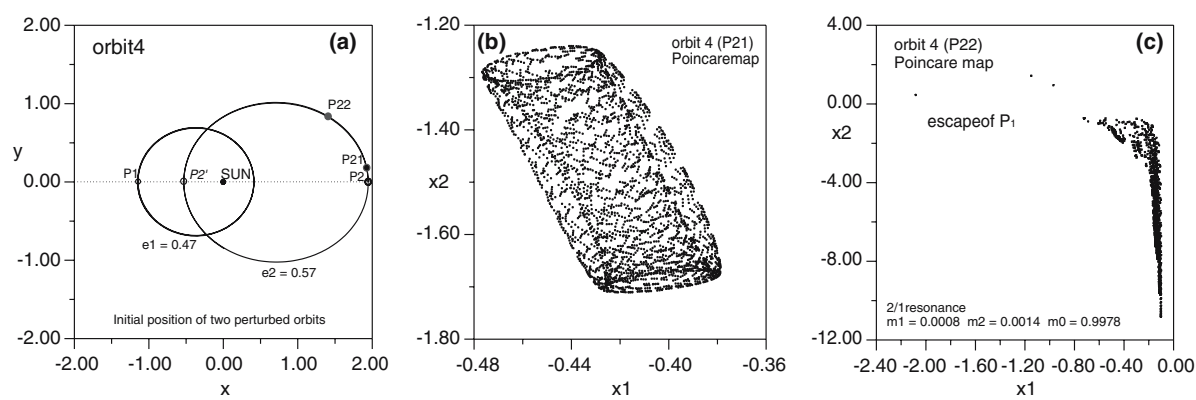

Fig. 14 (a) The stable orbit 4 and the two shifted positions, $P_{21}$ and $P_{22}$ of $P_{2}$ on its orbit. (b) The Poincaré map: $P_{2}$ at $P_{21}$, resulting to stable librations. (c) The Poincaré map: $P_{2}$ at $P_{22}$, resulting to chaotic motion and disruption of the system

at aphelion) are unstable. Note that the stable configurations are close to a stable family of periodic orbits.

\section{Discussion}

From all the previous examples it is clear that the position and the stability character of the periodic orbits plays an essential role in defining the structure of the phase space. A real extrasolar planetary system can exist in nature, if it lies in that region of the phase space which is close to a stable branch of a family of periodic orbits. We remark that all families of periodic orbits with non zero eccentricities are resonant, corresponding to a mean motion resonance between the planets. This means that resonances play an important role on the stability of a planetary system with large eccentricities, when the two planets are close to each other, so that their gravitational interaction is important. A phase protection mechanism appears in resonant cases, for some phases (alignment or antialignment, position of the planets at perihelion or aphelion), in such a way that we can have stable planetary systems even with large eccentricities and intersecting planetary orbits. Thus, all other things being the same (masses, eccentricities, and semimajor axes), the phase plays an important role on the stability of a resonant orbit. In addition, comparing Figs. 14 and 16, that correspond to two orbits with the same phase, one stable and one unstable, respectively, we have an indication that the increase of the planetary eccentricities may stabilize the system, because it makes the planets to avoid close encounters. 

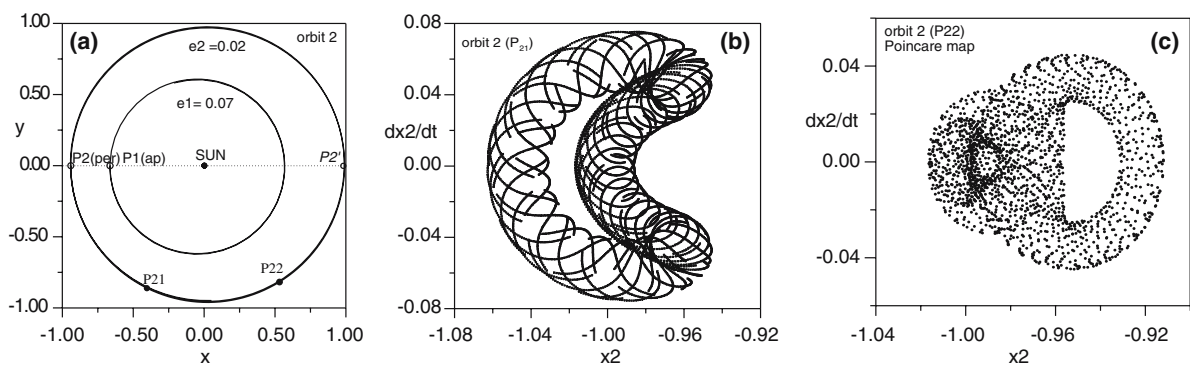

Fig. 15 (a) The stable orbit 2 and the two shifted positions, $P_{21}$ and $P_{22}$ of $P_{2}$ on its orbit. (b) The Poincaré map: $P_{2}$ at $P_{21}$, resulting to stable librations. (c) The Poincaré map: $P_{2}$ at $P_{22}$, resulting also to stable librations
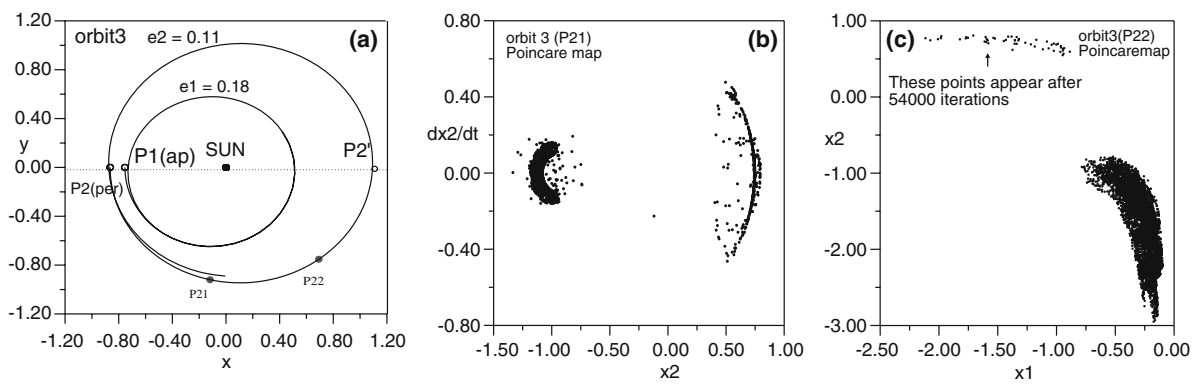

Fig. 16 (a) The unstable orbit 3 and the two shifted positions, $P_{21}$ and $P_{22}$ of $P_{2}$ on its orbit. (b) $P_{2}$ at $P_{21}$, resulting to chaotic motion. For a long time the motion is trapped on a 'torus' (the dark region, with the shape of the torus of figure a but later it deviates from this torus and the motion is chaotic. (c) $P_{2}$ at $P_{22}$, resulting to chaotic motion and disruption of the system

The resonances are not the only cases where stable motion exists. All non resonant periodic orbits on the circular family of periodic orbits are stable (with the exception of a small region close to the $3 / 1,5 / 3, \ldots$ resonances) and consequently stable librations are expected in their vicinity. In this case however, the planetary eccentricities are small.

Another factor that affects the topology of the phase space, and consequently the stability regions for a planetary system, is the ratio $m_{1} / m_{2}$ of the planetary masses and also the sum $m_{1}+m_{2}$ of the planetary masses, because it affects the appearance or not of families of periodic orbits and also the stability regions on a family of periodic orbits. As an example, consider the family 1 at the 2:1 resonance (Fig. 11). For $m_{1}<m_{2}$, the whole family is stable, but when the ratio of the planetary masses changes, $m_{1}>m_{2}$, an unstable region appears (Fig. 10) and we have a bifurcation of a new family of asymmetric periodic orbits (the critical mass ratio beyond which an unstable region appears on the family is $m_{1} / m_{2}=0.97$ ). This changes the topology and the stability properties of the phase space.

The total mass of the planetary masses, which is a measure of the perturbation, plays also an important role in some resonances, and in particular, in the resonances $3: 1,5: 3, \ldots$ As we showed in Sect. 4.4, we have a bifurcation of two families of elliptic orbits if the sum $m_{1}+m_{2}$ is small, but we have four elliptic families if this sum is larger. This means that the topology of the phase space, and consequently the dynamical properties of the system depend critically on the sum $m_{1}+m_{2}$ of the masses close to the 3:1 resonance.

As an example to show how the knowledge of the resonant structure of the phase space may help us to locate the positions of stable planetary systems, we present in Fig. 18a the families of periodic orbits for the masses of the system Gliese 876. The stable family 1 exists, 

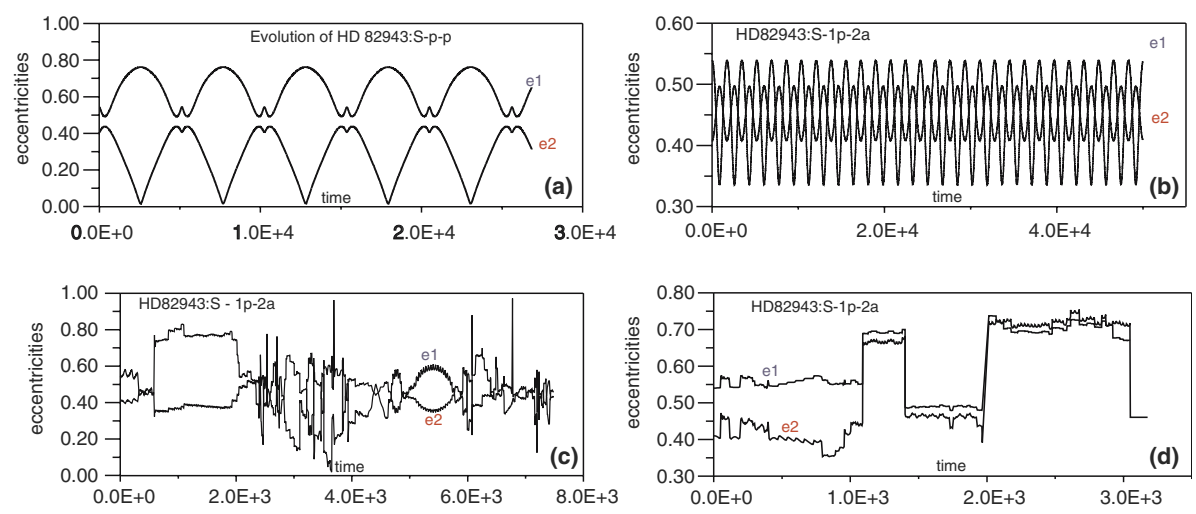

Fig. 17 The evolution of the eccentricities of the system HD 82943 for the four different positions shown in Fig. 11. (a) alignment, $P_{1}, P_{2}$ at perihelion: ordered motion. (b) antialignment, $P_{1}$ at aphelion, $P_{2}$ at perihelion: ordered motion. (c) alignment, $P_{1}, P_{2}$ at apocenter: chaotic motion. (d) antialignment, $P_{1}$ at perihelion, $P_{2}$ at aphelion: chaotic motion
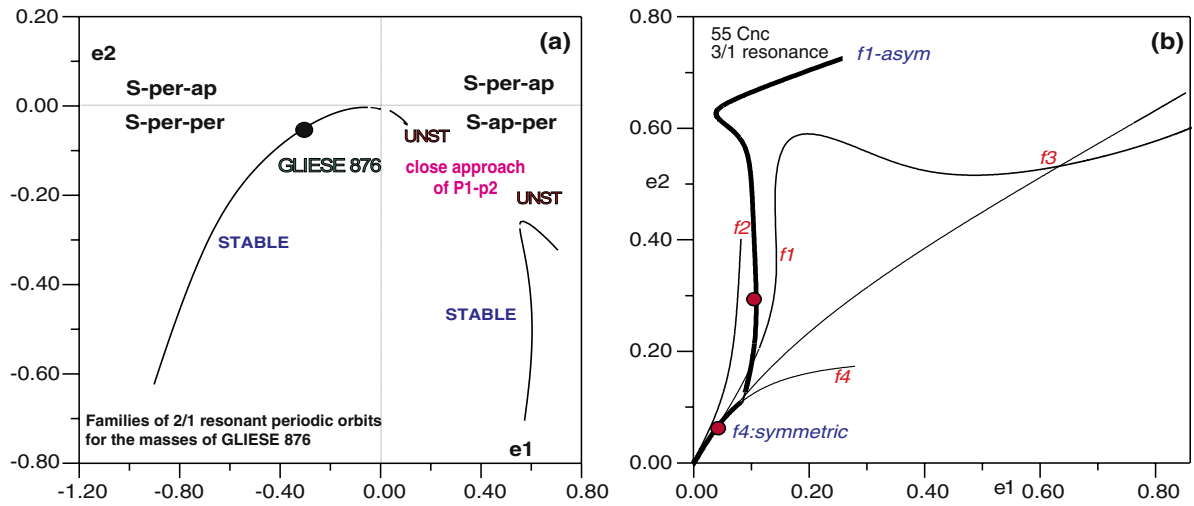

Fig. 18 (a) The 2:1 resonant families for the masses of Gliese 876, and the position of the observed system on the family 1. (b) The four different families, $f 1, f 2, f 3, f 4$ of $3: 1$ resonant periodic orbits. Only one of them, $f 4$, denoted by the thicker line, is stable up to a point. A new, stable asymmetric family, $f 1$-asym, bifurcates from this critical point. The planetary system, under migration, moves on the stable part of $f 4$ and then changes route and follows the family $f 1$-asym

as in the case of HD 82943 (Fig. 11), and we see that the elements of the observed system (from the web page of Schneider 2006), $a_{1}=0.13 \mathrm{AU}, a_{2}=0.21 \mathrm{AU}, e_{1}=0.27$, and $e_{2}=0.025$ lie almost exactly on the stable family 1, as it is shown in Fig. 18a.

Finally, we remark that the knowledge of the resonant structure of the phase space is useful in interpreting the results on the evolution of a planetary system under migration, when dissipative forces act. From the work on migration by Ferraz-Mello et al. (2003), Peale and Lee (2002), Lee and Peale (2003), and Lee (2004), we see that when a planetary system starts from an arbitrary position, it is trapped on a stable family of periodic orbits, and as the migration continues, it moves on the stable family. It is worth noting that if, as the system evolves on a stable family, there is a change from stability to instability, from which another stable family bifurcates, the system changes route and follows the new, stable, family. This 
is shown in Fig. 18b, for a system close to the 3:1 resonance, studied by Ferraz-Mello et al. (2003). There are four different families of resonant symmetric periodic orbits, $f 1, f 2, f 3$, and $f 4$ (Voyatzis and Hadjidemetriou 2006), and only one family, $f 4$, is stable at its lower part. The system is trapped on this stable branch and moves up to the point where the family becomes unstable. From that point on, we have a bifurcation of a stable asymmetric 3:1 resonant family of periodic orbits, family $f 1$-asym, and the system continues to migrate on this latter stable family.

From all the above, it is clear that the knowledge of the resonant structure of the phase space, as determined by the network of families of periodic orbits, is very useful in understanding the dynamics of a planetary system and interpreting the results obtained by different methods.

Acknowledgements I thank Dr. C. Beauge whose remarks helped me improve the presentation of the paper. This work was supported by the research program Pythagoras II, Nr.80831 of the Greek Ministry of Education and the E.U.

\section{References}

Beauge, C., Ferraz-Mello, S., Michtchenko, T.: Extrasolar planets in mean-motion resonance: apses alignment and asymmetric stationary solutions. ApJ 593, 1124 (2003)

Beauge, C., Michtchenko, T., Ferraz-Mello, S.: Stationary solutions in resonant extrasolar systems. MNRAS, 365, 1160-1170 (2006)

Beauge, C., Callegari, N., Ferraz-Mello, S., Michtchenko, T.: Resonances and stability of extra-solar planetary systems. In: Knezevic Z., Milani A. (eds.) Dynamics of Populations of Planetary Systems. Cambridge University Press, Cambridge, p. 3

Ferraz-Mello, S., Beaugé, C., Michtchenko T.: Evolution of migrating planet pairs in resonance, Cel. Mech. Dyn. Astr. 87, 99-112 (2003)

Fischer, D., Marcy, G., Buttler, P., Laughlin, G., Vogt, S.: A second planet orbiting 47 UMa. ApJ 564, 1028 (2002)

Gozdziewski, K., Bois, E., Maciejewski, A.: Global dynamics of the Gliese 876 planetary system. MNRAS 332, 839 (2002)

Hadjidemetriou, J.D.: The continuation of periodic orbits from the restricted to the general three-body problem. Cel. Mech. Dyn. Astr. 12, 155-174 (1975)

Hadjidemetriou, J.D.: Families of periodic planetary type orbits in the three-body problem and their stability. Astrophys. Sp. Sci. 40, 201-224 (1976)

Hadjidemetriou, J.D.: On the relation between resonance and instability in planetary systems. Cel. Mech. Dyn. Astr. 27, 305-322 (1982)

Hadjidemetriou, J.D.: Resonant periodic motion and the stability of extrasolar planetary systems. Cel. Mech. Dyn. Astr. 83, 141-154 (2002)

Hadjidemetriou, J.D.: Periodic orbits in gravitational systems. In: Proceedings of the Cortina meeting 2004, 2006

Hadjidemetriou, J.D., Psychoyos, D.: Dynamics of extrasolar planetary systems: 2/1 resonant motion. In: G. Contopoulos and N. Voglis (eds.), Lecture Notes in Physics: Galaxies and Chaos, Vol. 626, pp. 412 432, Springer-Verlag, Berlin (2003)

Israelian, G., Santos, N., Mayor, M., Rebolo, R.: Evidence for planet engulfment by the star HD82943. Nature 411, 163 (2001)

Ji, J., Liu, L., Kinoshita, H., Zhou, J., Nakai, H., Li, G.: The librating companions in HD 37124, HD 12661, HD 82943, 47 Uma and GJ 876: alignment or antialignment? ApJ 591, L57-L60 (2003a)

Ji, J., Kinoshita, H., Liu, L., Li, G., Nakai, H.: The apsidal antialignment of the HD 82943 system. Cel. Mech. Dyn. Astron. 87, 113-120 (2003b)

Lee, M.H., Peale, S.: Dynamic and origin of the 2:1 orbital resonances of the GJ 876 planets. ApJ 567, 596-609 (2002)

Lee, M.H., Peale, S.J.: Extrasolar planets and mean motion resonances. In Deming, D., Seager, S. (eds) Scientific Frontiers in Research of Extrasolar planets. ASP, 197 (2003)

Lee, M.H.: Diversity and origin of 2:1 orbital resonance in extrasolar planetary systems. ApJ 611, 517 (2004)

Malhotra, R.: A dynamical mechanism for establishing apsidal resonance. ApJ 575, L33-36 (2002) 
Marcy, G., Butler, P., Fischer, D., Vogt, S., Lissauer, J., Rivera, E.: A Pair of resonant planets orbiting GJ 876, ApJ 556, 296 (2001)

Marcy, G.W., Butler, R.P., Fischer, D.A., Laughlin, G., Vogt, S.S., Henry, G.W., Pourbaix, D.: A planet at 5AU around 55Cnc. ApJ 581, 1375-1388 (2002)

Mayor, M., Udry, S., Naef, D., Pepe, F., Queloz, D., Santos, N.C., Burnet, M.: The CORALIE survey for southern extra-solar planets. XII. Orbital solutions for 16 extra-solar planets discovered with CORALIE. A\&A 415, 291 (2004)

Nelson, R., Papaloizou, J.C.B.: Possible commensurabilities among pairs of extrasolar planets. MNRAS 333, 25-30 (2002)

Peale, S., Lee, M.: Extrasolar planets and the 2:1 orbital resonances, In DDA 33rd Meeting, BAAS 34, 933 (2002)

Psychoyos, D., Hadjidemetriou, J.D.: Dynamics of 2/1 resonant extrasolar systems. Application to HD82943 and Gliese876. Cel. Mech. Dyn. Astr. 92, 135-156 (2005)

Rivera, E.J., Lissauer, J.J.: Dynamical models of the resonant pair of planets orbiting the star GJ 876, ApJ 558, 392-402 (2001)

Schneider, J.: http://www.obspm.fr/encycl/catalog.html, (2006)

Voyatzis, G., Hadjidemetriou, H.D.: Symmetric and asymmetric librations in planetary and satellite systems at the 2/1 resonance. Cel. Mech. Dyn. Astr. 93, 263-294 (2005)

Voyatzis, G., Hadjidemetriou, H.D.: Symmetric and asymmetric 3:1 resonant periodic orbits: an application to the 55Cnc extra-solar system. Cel. Mech. Dyn. Astr. this issue (2006) 